\title{
Paeonol suppresses invasion, migration, and epithelial-to-mesenchymal transition in colorectal cancer cells through inhibition of COX-2 and PGE2
}

\section{Ming Li}

Renmin Hospital of Wuhan University

\section{Shi-yun Tan}

Renmin Hospital of Wuhan University

Yuan-jie Yu (D 2009283020197@whu.edu.cn )

Renmin Hospital of Wuhan University https://orcid.org/0000-0003-1814-9711

\section{Research}

Keywords: paeonol, colorectal cancer, EMT, cyclooxygenase-2, prostaglandin E2

Posted Date: March 10th, 2020

DOI: https://doi.org/10.21203/rs.3.rs-16564/v1

License: (c) (1) This work is licensed under a Creative Commons Attribution 4.0 International License.

Read Full License 


\section{Abstract}

Purpose: To observe the effects of paeonol on the invasion and migration of LoVo colorectal cancer cells, and investigate its possible mechanisms.

Materials and Methods: Cell transwell assay and wound-healing assay were applied, and the results suggested that paeonol could significantly inhibit the invasion and migration abilities of LoVo cells, which was associated with a reduction in COX-2 expression and PGE2 synthesis. Treatment with the selective COX-2 inhibitor, celecoxib, or transient transfection of colorectal cancer cells with COX-2 siRNA, also inhibited cell invasion and migration.

Results: The invasion and migration capacity were evaluated in LoVo cells by transwell assay and wound-healing in vitro. Compared with the control group, the invasion cells through Matrigel and the wound-healing rate were significantly decreased after treated with paeonol for $24 \mathrm{~h}$. Paeonol treatment downregulated the expression of MMP-9 and downregulated the COX-2 expression and PGE2 synthesis in LoVo cells. Paeonol could up-regulate the expression of epithelial marker E-cadherin while down-regulate the expressions of mesenchymal markers, Fibronectin and Vimentin. Paeonol inhibited PI3K-Akt and MAPK-ERK pathways in LoVo cells. Celecoxib treatment significantly decreased the cells penetrating the matrigel in a dose-dependent manner. siRNA knockdown of COX-2 leaded to inhibition of cell invasion in LoVo cells. Knockdown of COX-2 increased the expression of E-cadherin, whereas the expressions of Fibronectin and Vimentin were downregulated.

Conclusion: Paeonol may inhibit PI3K-Akt and MAPK-ERK pathways through suppressing of the COX-2 expression and PGE2 synthesis, thus inhibiting the cell invasion, migration, and EMT in LoVo cells.

\section{Background}

Colorectal cancer (CRC), a common malignancy of the digestive system, is a serious public health problem. CRC is the second leading cause of death from cancer in the developed world. What's worse, the CRC death rate is still increasing in many developing countries. ${ }^{1-3}$ Although a rapid progress has been achieved about the treatment and diagnosis in recent years, the prognosis for CRC remain poor for patients with metastasis. Therefore, the development of novel agents to block metastasis is an important area of study for addressing CRC.

Previous studies have demonstrated that cyclooxygenase-2 (COX-2) is constitutively overexpressed in a variety of malignancies, including colorectal cancer, breast cancer, gastric cancer, bladder cancer and non-small cell lung cancer (NSCLC). ${ }^{4,5}$ Deregulation of COX-2 expression leads to an increased abundance of prostaglandin $\mathrm{E}_{2}\left(\mathrm{PGE}_{2}\right)$, through which COX-2 contributes to carcinogenesis, progression, invasion, metastasis. ${ }^{6,7}$ It has been confirmed that COX-2 inhibitors could effectively decrease the incidences of gastric, colon, lung, breast and prostate cancer to some extent. ${ }^{8,9}$ Therefore, COX-2 is considered as a promising target for cancer therapy. 
Paeonol (2-hydroxy-4-methoxyacetophenone), a major active extract from the root bark of Paeonia suffruticosa Andrews, possesses a wide range of biologic and pharmacologic activities, including antiinflammatory ${ }^{10,11}$ anti-allergic, ${ }^{12}$ Anti-oxidative, ${ }^{13}$ and anti-platelet aggregation. ${ }^{14}$ The dosage forms of paeonol for clinical application approved by China Food and Drug Administration include injection, tablet and ointment. It can be used in treating inflammation/pain-related indications such as fever, headache, neuralgia, muscle pain and rheumatoid arthritis, as well as various skin diseases such as dermatitis, eczema, mosquito and bedbug bites. ${ }^{15}$ Recently, the antitumor effect of paeonol has garnered considerable attention. Unlike cytotoxic drugs, paeonol has shown cardioprotective, ${ }^{16}$ hepaticprotective, ${ }^{17-19}$ renal protective ${ }^{20}$ and neuroprotective effects. ${ }^{21}$ Paeonol has been shown to exhibit antitumor activities on gastric, ${ }^{22,23}$ prostate, ${ }^{24}$ breast, ${ }^{25}$ hepar, $^{26}$ ovarian $^{27}$ and esophageal cancer cells. ${ }^{28}$ Our previous study also revealed that paeonol exhibited the inhibiting effects on colorectal cancer lines (HCT116, SW620 and LoVo), especially in the LoVo cells. ${ }^{29}$ Here, the present study aimed to further investigate the effects of paeonol on the invasion and migration, and explore the underlying mechanisms in colorectal cancer cells.

\section{Materials And Methods}

Cell culture.

Colorectal cancer cell line LoVo was provided by the Cell Bank of the Shanghai Institute of Biochemistry and Cell Biology, Chinese Academy of Sciences (Shanghai, China). Colorectal cancer cells were incubated in DMEM/F-12 (Gibco, Carlsbad, CA, USA) containing 10\% fetal bovine serum (FBS; Gibco, Life Technologies, Carlsbad, CA), $100 \mathrm{U} / \mathrm{ml}$ penicillin and $100 \mu \mathrm{g} / \mathrm{ml}$ streptomycin in $5 \% \mathrm{CO}_{2}$ at $37{ }^{\circ} \mathrm{C}$. Cells in logarithmic growth phase were chosen for experiment.

Transwell assay for cell invasion.

For the cell-invasion test, 24-well transwells (pore size = $8 \mathrm{~mm}$; Corning Incorporated, Corning, NY, USA) were coated with Matrigel $(1 \mathrm{mg} / \mathrm{mL}$ ) according to the manufacturer's (Becton Dickinson) instructions. LoVo cells were suspended in the upper chamber $\left(2 \times 10^{5}\right.$ cells/well $)$ of serum-free medium, and the lower chamber was $500 \mu \mathrm{L}$ DMEM/F-12 medium containing 10\% FBS. After 24 hrs of incubation with the indicated concentrations of paeonol (99\% purity, Natura Pharmaceutical Co., Ltd. Zhejiang, China) or Celecoxib (Sigma-Aldrich, St. Louis, MO, USA), the cells of upper chamber were wiped clean by a cotton swab. Subsequently, the cells invading the lower chamber were fixed using $95 \%$ ethanol for 20 minutes, and then stained by $0.1 \%$ crystal purple for 10 minutes.

Wound-healing assay for cell migration.

Cell migration capacity was measured using a wound-healing assay. LoVo cells $\left(1 \times 10^{6}\right)$ were seeded in six-well plates and grew to form a confluent monolayer. a $200 \mu \mathrm{L}$ micropipette tip was used to create a wound. The detached cells were then removed by washing with PBS and incubated with or without 
paeonol for $24 \mathrm{hrs}$. The photomicrographs were taken under the inverted microscope at 0 , and $24 \mathrm{hrs}$ of the same wound areas, and experiments were carried out in triplicate.

Determination of $\mathrm{PGE}_{2}$ production.

The level of $\mathrm{PGE}_{2}$ in the cell culture supernatant was performed using the $\mathrm{PGE}_{2}$ ELISA kit (Thermo Scientific, Rockford, IL, USA) according to the manufacturer's protocol. And the experiments were carried out in triplicate.

COX-2 siRNA synthesis and transfection.

LoVo cells $\left(2 \times 10^{5}\right.$ in $2 \mathrm{ml}$ of DMEM/F-12 without antibiotics) were seeded in six-well plates. After $24 \mathrm{~h}$, the COX-2 siRNA (Shanghai GenePharma Co., Ltd. Shanghai, China) mix with Lipofectamine 2000 (Invitrogen, Carlsbad, CA, USA) was overlaid on the cells according to the manufacturer's instructions.

Western blot assay.

The cells were lysed with RIPA buffer supplemented with protease inhibitor. Total protein extract was separated on a $12.5 \%$ sodium dodecyl sulfate-polyacrylamide (SDS-PAGE) gel, and then transferred to nitrocellulose membranes using a wet transfer system. The membrane, after blocked by $5 \%$ fat-free milk, was incubated with primary antibodies: COX-2 (1:1000, Cell Signaling Technology), MMP-9 (1:1000, Cell Signaling Technology), E-cadherin(1:1000, Cell Signaling Technology), Vimentin (1:1000, Cell Signaling Technology), Fibronectin(1:1000, Cell Signaling Technology), Akt (1:1000, Cell Signaling Technology), pAkt(1:1000, Cell Signaling Technology), ERK (1:1000, Cell Signaling Technology), p-ERK(1:2000, Cell Signaling Technology), and $\beta$-actin (1:1000, Cell Signaling Technology) at $4{ }^{\circ} \mathrm{C}$ for 12 hours. Following that, PVDF was then rinsed with TBST solution and incubated at room temperature for 1 hour with the secondary antibodies (Cell Signaling Technology) conjugated to HRP. The automatic developing instrument (ChemiDocXRS imaging system) was adopted to develop and the gray value was calculated.

Statistical analysis.

All data were presented as Mean \pm SD. GraphPad Prism 7.0 was adopted for statistical analysis of data. Student's t-test was used for comparison of the values between two groups. $\mathrm{P}<0.05$ indicated statistical significance.

\section{Results}

Paeonol inhibited cell invasion and migration in LoVo cells.

The invasion and migration capacity were evaluated in LoVo cells by transwell assay and wound-healing in vitro. Compared with the control group, the invasion cells through Matrigel and the wound-healing rate were significantly decreased after treated with paeonol for $24 \mathrm{~h}$ (Fig. 1A-D). Meanwhile, paeonol 
treatment downregulated the expression of MMP-9, which could degrade extracellular matrix and destroy basilar membrane, therefore promotes tumor cell metastasis (Fig. 1E-F).

Paeonol downregulated the COX-2 expression and PGE2 synthesis in LoVo cells.

To investigate whether $\mathrm{COX}-2$ and $\mathrm{PGE}_{2}$ are involved in the antitumor effect of paeonol, the protein level of COX-2 was assessed by western blot, and the level of $\mathrm{PGE}_{2}$ in the cell culture supernatant was performed using the $\mathrm{PGE}_{2}$ ELISA kit. The results showed that Paeonol treatment decreased COX-2 expression and PGE2 synthesis in LoVo cells in a dose-dependent manner (Fig. 2).

Paeonol reversed epithelial-mesenchymal transition (EMT) in LoVo cells.

It is demonstrated that Epithelial-mesenchymal transition (EMT) plays a key role in initiating cancer metastasis. To investigate whether EMT is also involved in the inhibiting effects of paeonol on invasion and migration, the protein levels of EMT biomarkers, E-cadherin, Fibronectin and Vimentin, were assessed by western blot. Paeonol could up-regulate the expression of epithelial marker E-cadherin while downregulate the expressions of mesenchymal markers, Fibronectin and Vimentin (Fig. 3).

Paeonol inhibited PI3K-Akt and MAPK-ERK pathways in LoVo cells.

PI3K-Akt and MAPK-ERK pathways are correlated with cancer invasion and EMT. To ascertain if the PI3KAkt and MAPK-ERK pathways are involved in the antitumor effect of paeonol, the protein levels of Akt, pAkt, ERK and p-ERK in LoVo cells was examined using western blot analysis. Paeonol treatment decreased expression of p-Akt and p-ERK in LoVo cells in a dose-dependent manner (Fig. 4).

Celecoxib, a selective COX-2 inhibitor, inhibited invasion in LoVo cells.

The invasion capacity of LoVo cells was evaluated by transwell assay after treatment with various concentrations of celecoxib $(0,25,50$ and $100 \mu \mathrm{mol} / \mathrm{l})$, a well-known inhibitor of COX-2, for $24 \mathrm{~h}$. The data showed that celecoxib treatment significantly decreased the cells penetrating the matrigel in a dosedependent manner (Fig. 5).

siRNA knockdown of COX-2 leaded to inhibition of cell invasion in LoVo cells.

We further verified the role of COX-2 in cell invasion through siRNA knockdown of COX-2 in the colorectal cells and investigated whether it would lead to the inhibition of cell invasion. The data showed that the transfection with COX-2 siRNA resulted in a significant inhibition in the cell invasion in LoVo cells after $24 \mathrm{~h}$ as compared to that of the control siRNA-transfected LoVo cells (Fig. 6A-B). And downregulation of COX-2 decreased the expressions of MMP-9 (Fig. 6C).

siRNA knockdown of COX-2 regulated EMT and inhibited PI3K-Akt and MAPK-ERK pathways in LoVo cells. 
Knockdown of COX-2 in LoVo cells inhibited cell invasion, so we further aimed to investigate the effects of COX-2 on EMT, PI3K-Akt and MAPK-ERK pathways. First, the expression of EMT markers was analyzed in COX-2 knockdown LoVo cells and the control, respectively. As shown in Fig. 7A, knockdown of COX-2 increased the expression of E-cadherin, whereas the expressions of Fibronectin and Vimentin were downregulated. Moreover, downregulation of COX-2 significantly decreased the expressions of $\mathrm{p}$-Akt and p-ERK in LoVo cells (Fig. 7B).

\section{Discussion}

A majority of $\mathrm{CRC}$ patients are diagnosed with advanced stage, resulting in poor prognosis. ${ }^{30}$ Metastatic dissemination is the leading cause of colorectal cancer-related deaths. Therefore, the development of novel, efficacious therapeutic agents to block metastasis is especially urgent.

In the present study, paeonol elicited dramatic inhibition of invasion and migration in LoVo cells as demonstrated by the transwell assay and wound-healing assay. Meanwhile, paeonol treatment decreased expression of MMP-9, which could degrade extracellular matrix and destroy basilar membrane, therefore promotes tumor cell metastasis.

Epithelial-to-mesenchymal transition (EMT), a biological process of epithelial phenotype transforming to interstitial phenotype because of extracellular factor stimulation, is the essential mechanism for initiating cancer invation and metastasis. ${ }^{31-33}$ During EMT, cancer cell polarity, intercellular tight junction and adhesion junction are gradually disappearing. Moreover, the expression of epithelial marker, E-cadherin, was up-regulated and the expressions of mesenchymal markers, such as Fibronectin, N-cadherin and Vimentin, were down-regulated in tumor cells. ${ }^{34}$ Meanwhile, the expressions of MMPs in tumor cells are up-regulated. ${ }^{35}$ The results demonstrated that paeonol treatment of LoVo cells showed the suppression of mesenchymal markers Fibronectin and Vimentin, and restored the levels of epithelial marker Ecadherin, which suggest that paeonol could reverse the EMT process in LoVo cells, thus inhibiting the cell invasion and migration abilities.

COX-2 induced the synthesis of prostaglandins, mainly $\mathrm{PGE}_{2}$, promotes tumor cell proliferation, invasion, angiogenesis, and metastasis. $\mathrm{COX}-2$ and $\mathrm{PGE}_{2}$ have also been verified to be associated with $\mathrm{EMT}^{36}$ Thus, we further explored the effects of paeonol on the expression of COX-2 and synthesis of $\mathrm{PGE}_{2}$ in LoVo cells. The results showed that treated with paeonol leaded to a significant reduction in the expression of COX-2 and synthesis of $\mathrm{PGE}_{2}$ in the LoVo cells. And treatment with celecoxib, a well-known inhibitor of COX-2, also resulted in a reduction in the cell invasion in LoVo cells. Similar effects were observed when the LoVo cells were transfected with COX-2 siRNA. Moreover, inhibition of COX-2 expression in LoVo cells by a specific silencer RNA resulted in the upregulation of an epithelial marker Ecadherin, in the downregulation of mesenchymal markers Fibronectin and Vimentin. These findings indicate that paeonol inhibits cell invasion and migration by reducing the COX-2 expression of COX-2 and $\mathrm{PGE}_{2}$ synthesis, which leads to suppression of EMT in LoVo cells. 
PI3K-Akt and MAPK-ERK pathways are are pivotal signaling pathways during tumor migration, invasion, metastasis and EMT, and COX-2 expression was also correlated with PI3K-Akt and MAPK-ERK

pathways. ${ }^{37-39}$ Therefore, we sought to determine whether PI3K-Akt and MAPK-ERK pathways were involved in the antitumor effect of paeonol. In accordance with our expectation, paeonol treatment decreased expression of p-Akt and p-ERK in LoVo cells. And inhibition of COX-2 expression in LoVo cells by a specific silencer RNA also decreased the expressions of p-Akt and p-ERK in LoVo cells. These results indicate that paeonol may inhibit PI3K-Akt and MAPK-ERK pathways by suppression of the COX-2 expression and $\mathrm{PGE}_{2}$ synthesis, thus inhibiting the cell migration, invasion, and EMT in LoVo cells.

\section{Conclusion}

The results demonstrated that paeonol could inhibit invasion, migration, and EMT of CRC cells through inhibition of COX-2 expression and $\mathrm{PGE}_{2}$ synthesis. These results may provide new insights into the potential mechanisms of paeonol on invasion, migration and EMT in CRC cells, and the effects of paeonol on CRC metastasis in vivo need further investigated.

\section{Declarations}

Acknowledgements

Not applicable.

Funding

The present study was supported by grants from the Natural Science Foundation of Hubei Province (2019CFB142).

Authors' contributions

Ming Li and Shiyun Tan designed the research; Ming Li and Yuanjie Yu performed the research; Ming Li and Yuanjie Yu analyzed the data; Ming Li wrote the paper.

Ethics approval and consent to participate: Ethics approval and consent to participate acquired.

Consent for publication: All authors consent for publication in European Journal of Medical Research

Availability of data and materials: Avaailable under certain requirements

Competing interests: The authors declare that they have no competing interests.

\section{References}


1. Wolf AMD, Fontham ETH, Church TR, Flowers CR, Guerra CE, LaMonte SJ, et al. Colorectal cancer screening for average-risk adults: 2018 guideline update from the American Cancer Society. CA Cancer J Clin. 2018;68:250-281.

2. Siegel RL, Miller KD, Jemal A. Cancer statistics, 2018. CA Cancer J Clin. 2018;68:7-30.

3. Ferlay J, Soerjomataram I, Dikshit R, Eser S, Mathers C, Rebelo M, et al. Cancer incidence and mortality worldwide: sources, methods and major patterns in GLOBOCAN 2012. Int J Cancer. 2015; 136: E359-386.

4. Hashemi Goradel N, Najafi M, Salehi E, Farhood B, Mortezaee K. Cyclooxygenase-2 in cancer: A review. J Cell Physiol. 2019;234:5683-5699.

5. Desai SJ, Prickril B, Rasooly A. Mechanisms of Phytonutrient Modulation of Cyclooxygenase-2 (COX2) and Inflammation Related to Cancer. Nutr Cancer. 2018;70:350-375.

6. Karpisheh V, Nikkhoo A, Hojjat-Farsangi M, Namdar A, Azizi G, Ghalamfarsa G,et al. Prostaglandin $E_{2}$ as a potent therapeutic target for treatment of colon cancer. Prostaglandins Other Lipid Mediat. 2019;15:106338.

7. Sharma V, Bhatia P, Alam O, Javed Naim M, Nawaz F, Ahmad Sheikh A, et al. Recent advancement in the discovery and development of COX-2 inhibitors: Insight into biological activities and SAR studies (2008-2019). Bioorg Chem. 2019; 89: 103007.

8. Brizzolara A, Benelli R, Venè R, Barboro P, Poggi A, Tosetti F, et al. The ErbB family and androgen receptor signaling are targets of Celecoxib in prostate cancer. Cancer Letters. 2017;400: 9-17.

9. Shi L, Xu L, Wu C, Xue B, Jin X, Yang J, et al. Celecoxib-Induced Self-Assembly of Smart AlbuminDoxorubicin Conjugate for Enhanced Cancer Therapy. ACS App/ Mater Interfaces. 2018; 10: 85558565.

10. Zong SY, Pu YQ, Xu BL, Zhang T, Wang B. Study on the physicochemical properties and antiinflammatory effects of paeonol in rats with TNBS-induced ulcerative colitis. Int Immunopharmacol. 2017; 42: 32-38.

11. Lou Y, Wang C, Tang Q, Zheng W, Feng Z, Yu X, et al. Paeonol Inhibits IL-1 $\beta$-Induced Inflammation via PI3K/Akt/NF-KB Pathways: In Vivo and Vitro Studies. 2017; 40: 1698-1706.

12. Meng Y, Liu Z, Zhai C, Di T, Zhang L, Zhang L, et al. Paeonol inhibits the development of 1-chloro-2,4-dinitrobenzene-induced atopic dermatitis via mast and T cells in BALB/c mice. Mol Med Rep. 2019; 19: 3217-3229.

13. Jin X, Wang J, Xia ZM, Shang CH, Chao QL, Liu YR, et al. Anti-inflammatory and Anti-oxidative Activities of Paeonol and Its Metabolites Through Blocking MAPK/ERK/p38 Signaling Pathway. 2016; 39: 434-446.

14. Ye S, Liu X, Mao B, Yang L, Liu N, et al. Paeonol enhances thrombus recanalization by inducing vascular endothelial growth factor 165 via ERK1/2 MAPK signaling pathway. Mol Med Rep. 2016; 13: 4853-4858. 
15. Zhang L, Li DC, Liu LF. Paeonol: pharmacological effects and mechanisms of action. Int Immunopharmacol. 2019; 72: 413-421.

16. Wu J, Sun C, Wang R, Li J, Zhou M, Yan M, et al. Cardioprotective effect of paeonol against epirubicin-induced heart injury via regulating miR-1 and PI3K/AKT pathway. Chem Biol Interact. 2018; 286: 17-25.

17. Wu J, Xue X, Zhang B, Jiang W, Cao H, Wang R, et al. The protective effects of paeonol against epirubicin-induced hepatotoxicity in 4T1-tumor bearing mice via inhibition of the PI3K/Akt/NF-kB pathway. Chem Biol Interact. 2016; 244: 1-8.

18. Wu S, Liu L, Yang S, Kuang G, Yin X, Wang Y, et al. Paeonol alleviates CCl4-induced liver fibrosis through suppression of hepatic stellate cells activation via inhibiting the TGF- $\beta$ /Smad3 signaling. Immunopharmacol Immunotoxicol. 2019; 41:438-445.

19. Sun X, Wang P, Yao LP, Wang W, Gao YM, Zhang J, et al. Paeonol alleviated acute alcohol-induced liver injury via SIRT1/Nrf2/NF-KB signaling pathway. Environ Toxicol Pharmacol. 2018; 60: 110-117.

20. Wu J, Xu L, Sun C, Zhang B, Li J, Sun J, et al. Paeonol alleviates epirubicin-induced renal injury in mice by regulating Nrf2 and NF-KB pathways. Eur J Pharmacol. 2017; 795: 84-93.

21. Liu J, Feng L, Ma D, Zhang M, Gu J, Wang S, et al. Neuroprotective effect of paeonol on cognition deficits of diabetic encephalopathy in streptozotocin-induced diabetic rat. Neurosci Lett. 2013;549: 63-8.

22. Li N, Fan LL, Sun GP, Wan XA, Wang ZG, Wu Q, et al. Paeonol inhibits tumor growth in gastric cancer in vitro and in vivo. World J Gastroenterol. 2010; 16: 4483-90.

23. Fu J, Yu L, Luo J, Huo R, Zhu B. Paeonol induces the apoptosis of the SGC-7901 gastric cancer cell line by downregulating ERBB2 and inhibiting the NF-KB signaling pathway. Int J Mol Med. 2018;42:1473-1483.

24. Xu Y, Zhu JY, Lei ZM, Wan LJ, Zhu XW, Ye F, et al. Anti-proliferative effects of paeonol on human prostate cancer cell lines DU145 and PC-3. J Physiol Biochem. 2017;73: 157-165.

25. Ou Y, Li Q, Wang J, Li K, Zhou S. Antitumor and Apoptosis Induction Effects of Paeonol on Mice Bearing EMT6 Breast Carcinoma. Biomol Ther (Seoul). 2014;22: 341-346.

26. Fan L, Song B, Sun G, Ma T, Zhong F, Wei W.. Endoplasmic reticulum stress-induced resistance to doxorubicin is reversed by paeonol treatment in human hepatocellular carcinoma cells. PLoS One. 2013; 8: e62627.

27. Yin J, Wu N, Zeng F, Cheng C, Kang K, Yang H. Paeonol induces apoptosis in human ovarian cancer cells. Acta Histochem. 2013; 115: 835-839.

28. Ramachandhiran D, Vinothkumar V, Babukumar S. Paeonol exhibits anti-tumor effects by apoptotic and anti-inflammatory activities in 7,12-dimethylbenz(a)anthracene induced oral carcinogenesis. Biotech Histochem. 2019; 94: 10-25.

29. Li M, Tan SY, Wang XF. Paeonol exerts an anticancer effect on human colorectal cancer cells through inhibition of PGEX synthesis and COX-2 expression. Oncol Rep. 2014; 32:2845-2853. 
30. Arnold M, Sierra MS, Laversanne M, Soerjomataram I, Jemal A, Bray F, et al. Global patterns and trends in colorectal cancer incidence and mortality. 2017; 66: 683-691.

31. Brabletz T, Kalluri R, Nieto MA, Weinberg RA. EMT in cancer. Nat Rev Cancer 18: 128-134, 2018.

32. Cho ES, Kang HE, Kim NH, Yook JI. Therapeutic implications of cancer epithelial-mesenchymal transition (EMT). Arch Pharm Res 42: 14-24, 2019.

33. Mittal V. Epithelial Mesenchymal Transition in Tumor Metastasis. Annu Rev Pathol 13: 395-412, 2018.

34. Thiery JP. Epithelial-mesenchymal transitions in tumour progression. Nat Rev Cancer 2: 442-454, 2002.

35. Santibanez JF, Obradović H, Kukolj T, Krstić J. Transforming growth factor- $\beta$, matrix metalloproteinases, and urokinase-type plasminogen activator interaction in the cancer epithelial to mesenchymal transition. Dev Dyn 247: 382-395, 2018.

36. Wang YP, Wang QY, Li CH, Li XW. COX-2 inhibition by celecoxib in epithelial ovarian cancer attenuates E-cadherin suppression through reduced Snail nuclear translocation. Chem Biol Interact. 2018; 292: 24-29.

37. Majumder $M$, Xin X, Liu L, Tutunea-Fatan E, Rodriguez-Torres $M$, Vincent $K$, et al. COX-2 Induces Breast Cancer Stem Cells via EP4/PI3K/AKT/NOTCH/WNT Axis. Stem Cells. 2016; 34: 2290-305.

38. Xia Q, Hu Q, Wang H, Yang H, Gao F, Ren H, et al. Induction of COX-2-PGE2 synthesis by activation of the MAPK/ERK pathway contributes to neuronal death triggered by TDP-43-depleted microglia. Cell Death Dis. 2015; 6: e1702.

39. Lin L, Cheng K, He Z, Lin Q, Huang Y, Chen C, et al. A polysaccharide from Hedyotis diffusa interrupts metastatic potential of lung adenocarcinoma A549 cells by inhibiting EMT via EGFR/Akt/ERK signaling pathways. Int J Biol Macromol. 2019;129:706-714.

\section{Figures}

\section{Figure 1}

Paeonol inhibited cell invasion and migration in LoVo cells. (A) The cell invasion was measured by transwell assay. (B) Number of cells in each field was counted and averaged (\#P<0.05 vs. $0 \mathrm{mg} / \mathrm{l})$. (C) The cell migration was performed by wound-healing assay. (D) Migration of LoVo cells was quantified by measuring wound-closure areas before and after injury (\#P<0.05 vs. $0 \mathrm{mg} / \mathrm{l})$. (E) The expression of MMP9 was detected by Western blot. (F) Quantitative comparison of the expression level of MMP-9 among groups (\#P<0.05 vs. $0 \mathrm{mg} / \mathrm{l})$.

\section{Figure 2}


Paeonol reduced cyclooxygenase-2 (COX-2) expression and prostaglandin E2 (PGE2) synthesis in LoVo cells. (A) The expression of COX-2 was detected by Western blot. (B) Quantitative comparison of the expression level of COX-2 among groups (\#P<0.05 vs. $0 \mathrm{mg} / \mathrm{l})$. (C) The levels of PGE2 in the LoVo cell supernatant among groups ( $\# \mathrm{P}<0.05 \mathrm{vs} .0 \mathrm{mg} / \mathrm{l})$.

\section{Figure 3}

Paeonol reversed epithelial-mesenchymal transition (EMT) in LoVo cells. (A)The protein levels of Ecadherin, Fibronectin and Vimentin were assessed by western blot. (B) Quantitative comparison of the expression levels of E-cadherin, Fibronectin and Vimentin among groups ( $\# \mathrm{P}<0.05 \mathrm{vs} .0 \mathrm{mg} / \mathrm{l})$.

\section{Figure 4}

Paeonol inhibited PI3K-Akt and MAPK-ERK pathways in LoVo cells. (A) The protein levels of Akt, p-Akt, ERK and p-ERK were assessed by western blot. (B) Quantitative comparison of the expression levels of Akt, p-Akt, ERK and p-ERK among groups (\#P<0.05 vs. 0 mg/l).

\section{Figure 5}

Celecoxib inhibited invasion in LoVo cells. (A) The cell invasion was measured by transwell assay. (B) Number of cells in each field was counted and averaged (\#P<0.05 vs. $0 \mu \mathrm{mol} / \mathrm{l})$.

\section{Figure 6}

siRNA knockdown of cyclooxygenase-2 (COX-2) leads to inhibition of cell invasion in LoVo cells. (A) Cell invasion was measured by transwell invasion assay in Control siRNA-transfected LoVo cells and COX-2 siRNA-transfected LoVo cells. (B) Number of cells in each field was counted and averaged (\#P<0.05 vs. the control siRNA-transfected group). (C) The expression of MMP-9 in Control siRNA-transfected LoVo cells and COX-2 siRNA-transfected LoVo cells was assessed by western blot. (D) Quantitative comparison of the expression level of MMP-9 between the groups (\#P<0.05 vs. the control siRNA-transfected group).

\section{Figure 7}

siRNA knockdown of COX-2 regulated EMT and inhibited PI3K-Akt and MAPK-ERK pathways in LoVo cells. (A) and (C) LoVo cells were transfected with COX-2-siRNA or Control-siRNA, and the indicated proteins were measured by western blot. (B) and (D) Quantitative comparison of the expression level of the indicated proteins between the groups (\#P<0.05 vs. the control siRNA-transfected group). 